\title{
Safety and efficacy of liposomal amphotericin $B$ for the empirical therapy of invasive fungal infections in immunocompromised patients
}

REVIEW

\author{
Marisa H Miceli' \\ Pranatharthi Chandrasekar ${ }^{2}$ \\ 'Oakwood Hospital and Medical \\ Center, Dearborn, ${ }^{2}$ Division of \\ Infectious Diseases, Wayne State \\ University School of Medicine, \\ Detroit, MI, USA
}

This article was published in the following Dove Press journal:

Infection and Drug Resistance

10 January 2012

Number of times this article has been viewed

\begin{abstract}
Liposomal amphotericin B is a "true" liposomal formulation of amphotericin B with greatly reduced nephrotoxicity and minimal infusion-related toxicity. This broad spectrum polyene is well tolerated and effective against most invasive fungal infections. In view of the current limitations on diagnostic capability of invasive fungal infections, most clinicians are often compelled to use antifungal drugs in an empiric manner; liposomal amphotericin B continues to play an important role in the empiric management of invasive fungal infections, despite the recent availability of several other drugs in the azole and echinocandin classes.
\end{abstract}

Keywords: invasive fungal infections, immunocompromised hosts, empiric therapy, polyenes, efficacy and safety

\section{Introduction}

Invasive fungal infections (IFIs) have become a leading cause of morbidity and mortality among immunocompromised patients. ${ }^{1}$ The incidence of IFIs in these patient populations has increased dramatically over the past three decades. The most common fungal pathogens include Candida albicans and Aspergillus fumigatus and cryptococcus. However, uncommon pathogens such as non-albicans Candida spp, opportunistic yeast-like fungi (eg, Trichosporon and Rhodotorula spp), non-fumigatus Aspergillus spp, Mucorales and hyaline molds (eg, Fusarium and Scedosporium spp) are now emerging as not-so-uncommon opportunistic pathogens. ${ }^{2,3}$ Etiologic identification of fungal pathogens is frequently difficult, particularly in compromised hosts, since reliable, noninvasive diagnostic tests are presently not available; such situations often compel the clinician to use drugs in an empiric fashion. However, the suspected fungal pathogens are often less susceptible to many antifungal agents including the "broadlyactive" amphotericin B (AmB).$^{4-8}$ Thus, the choice of appropriate agents for empirical antifungal therapy has become a challenge; in the context of recently available, noninvasive diagnostic tests and newer antifungal drugs, the role of amphotericin B as a drug for empiric therapy has become less clear. This review will provide an overview of liposomal amphotericin B (L-AmB) and focus on efficacy studies as well as the safety and tolerability of this drug for the empiric treatment of IFIs.

\section{Management of IFIs in the immunocompromised host}

Multiple factors have contributed to the new epidemiologic trends of IFIs, notably a growing number of immunocompromised states (ie, cancer, hematologic stem cell 
and solid organ transplantation, major surgeries, acquired immune deficiency syndrome, the elderly, etc), the advent of potent immunosuppressant drugs and their widespread use, and the use of antifungal prophylaxis. ${ }^{9-12}$

In contrast to most bacterial infections, the diagnosis of IFIs is challenging. Delay in diagnosis leads to delayed initiation of appropriate antifungal therapy and, hence, increased mortality. In this setting, early and definitive diagnosis is critical in the management of IFIs. However, clinical presentation of IFIs is often nonspecific; moreover, signs and symptoms could be absent in the early stages of the disease. Fever without signs of localized infection is the most common clinical presentation. ${ }^{13}$ Fever is of particular concern in severely immunosuppressed patients such as those with profound and prolonged neutropenia following chemotherapy for cancer. Radiographic signs and laboratory tests such as galactomannan and 1,3-beta-D-glucan are used as surrogate markers for the diagnosis and management of IFIs. ${ }^{14,15}$

Prompt initiation of appropriate antifungal therapy of IFIs is crucial for optimal outcome. Current strategies for the management of IFIs include prophylactic, empiric, preemptive, and targeted therapy ${ }^{15,16}$ (Figure 1). Prophylactic strategy includes administration of a narrow- or broadspectrum antifungal drug (active against yeasts or yeasts and molds) to high-risk patient population before the onset of signs/symptoms of infection. Empirical antifungal therapy is often initiated when a microbiological diagnosis of IFI is unavailable in high-risk presently neutropenic patients presenting with persistent or recurring fevers despite 4-7 days of appropriate antibiotic therapy (duration of neutropenia expected to be more than 7 days).

Pre-emptive therapy is initiated when suggestive but nonspecific radiographic signs are present and/or laboratory tests are suggestive of IFIs, without microbiological or histopathological confirmation of IFI. Targeted therapy is initiated when there is microbiologically and/or histologically proven IFI. ${ }^{15,16}$

\section{Antifungal drugs}

There are three main classes of systemic antifungal drugs available for the treatment of IFIs: polyenes, azoles, and echinocandins. Azoles deplete ergosterol and compromise fungal cell-wall integrity by dose-dependent inhibition of cytochrome-P450-dependent $14 \alpha$-demethylase. ${ }^{17}$ Currently available systemic azoles include fluconazole, itraconazole, voriconazole, and posaconazole. Echinocandins are compounds that disrupt cell-wall synthesis by inhibiting the synthesis of 1,3-beta-D-glucan, which is a critical component of most fungal cell walls. ${ }^{18}$ Echinocandins include caspofungin, anidulafungin, and micafungin. Azoles and echinocandins are relatively safe and well tolerated. ${ }^{18,19}$

The polyenes include conventional amphotericinB(AmB-D) and lipid formulations of amphotericin $\mathrm{B}$ (amphotericin $\mathrm{B}$ lipid complex [ABLC], L-AmB, and amphotericin B colloi-

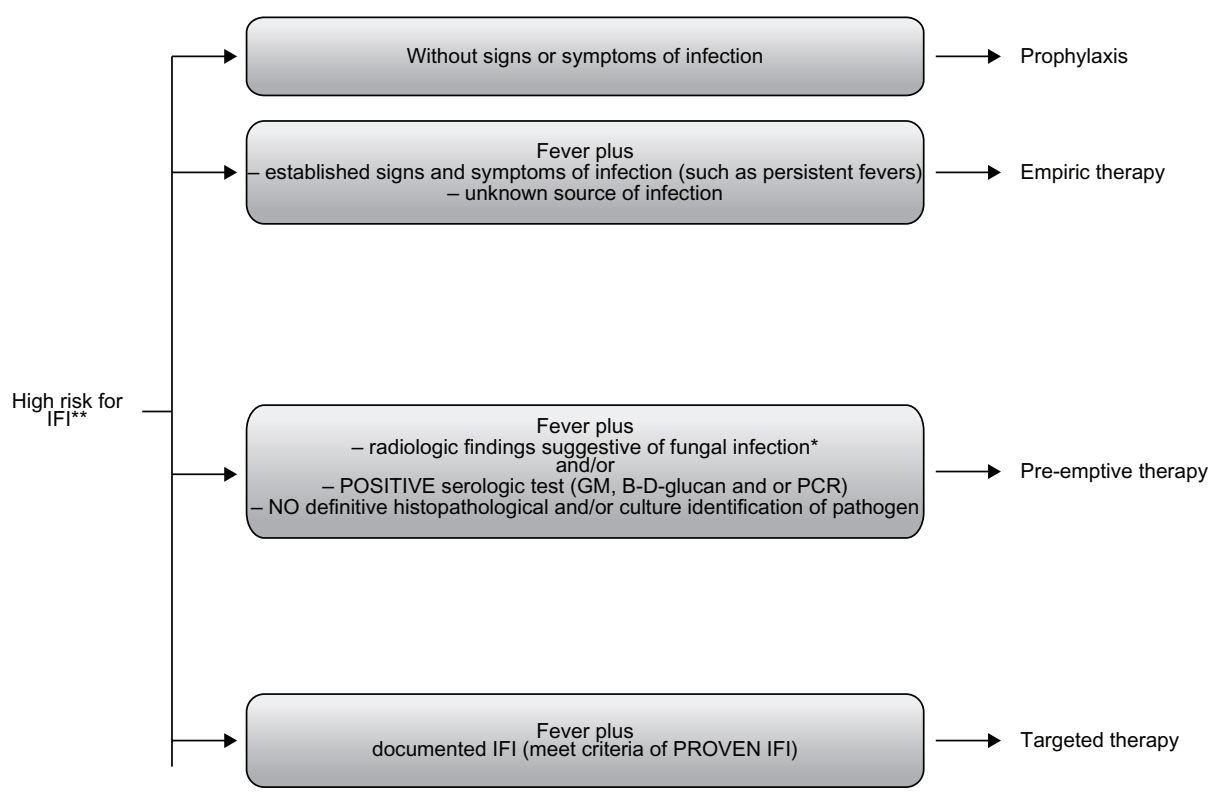

Figure I Current strategies for the management of IFI in high-risk patients.

Notes: *High-resolution computed tomography scan of the lungs showing new $\geq 1 \mathrm{~cm}$ single or multiple nodules with or without halo sign, lobar consolidation, wedge-shaped consolidative infarct; **classic example: patients with proglonged and profound neutropenia after intense chemotherapy for hematologic malignancy. Abbreviations: GM, serum Galactomannan; IFI, invasive fungal infection; PCR, polymerase chain reaction. 
dal dispersion $[\mathrm{ABCD}]$ formulations $) .{ }^{20} \mathrm{AmB}-\mathrm{D}$ binds with sterols, principally ergosterol in the fungal cell membrane. This binding is followed by increased leakage of intracellular ions out of the fungal cells resulting in osmotic disruption with increased membrane permeability and, ultimately, cell death. Polyenes also interfere with membrane-associated oxidative enzyme function, which is also lethal for the cell. ${ }^{20}$

Lipid formulations of amphotericin B were developed to decrease toxicity and improve the tolerability profile of AmB-D. Lipid formulations of amphotericin B have different structures. ABLC has a ribbon-like structure, $\mathrm{ABCD}$ is composed of disc-like structures, and L-AmB forms small uniform spherical lipid vesicles or liposomes containing amphotericin B. ${ }^{21}$ The three formulations produce tissue amphotericin B concentrations ranging from 90\% lower than, to $500 \%$ higher than the serum levels seen with AmB-D. Most consistently, reduced concentrations are seen in the kidney, thus accounting for the markedly lower nephrotoxicity. The doses of lipid forms of amphotericin B are higher than that of amphotericin B deoxycholate; such high but equipotent doses are better tolerated and there is also a clear reduction in the frequency and severity of nephrotoxicity.

\section{Spectrum of activity: amphotericin B}

Despite the differences in the structures of lipid formulations and AmB-D, their spectrum of activity is comparable. ${ }^{22}$ Amphotericin B is active against clinically relevant yeasts such as most Candida spp and Cryptococcus neoformans and molds, including most Aspergillus spp and Mucorales. AmB is also active against Histoplasma spp, Paracoccidiodes spp, Blastomyces spp, and Coccidioides spp. ${ }^{20}$

Several Candida species such as Candida lusitaniae, Candida guilliermondii, and Candida rugosa may be resistant to AmB. ${ }^{23-25}$ Also, Trichosporon spp, an infrequent cause of catheter-related fungemia, are resistant to AmB. ${ }^{26,27}$

Non-fumigatus Aspergillus spp, in particular Aspergillus terreus, Aspergillus flavus and Aspergillus nidulans, have demonstrated clinical resistance to AmB. ${ }^{28-30}$ Similarly, Aspergillus ustus is an uncommon clinical species that may have decreased susceptibility to AmB. ${ }^{31-33}$ However, Aspergillus lentulus, a recently described Aspergillus species, has demonstrated variable in vitro susceptibility to AmB. ${ }^{34,35}$ AmB has limited activity against Fusarium spp (especially Fusarium solani), although it has better activity as compared with other agents such as the older azoles (eg, Itraconazole) and echinocandins. ${ }^{8,36,37}$ Mucorales are susceptible to AmB - the drug has been the drug of choice for the treatment of mucormycosis; however, clinical response has been variable and the overall mortality of patients with mucormycosis has remained high, particularly in patients with persistent immunosuppression or poor source control. A newer azole, posaconazole is active against most Mucorales and appears to be promising for the treatment of this disease. ${ }^{8,38}$ Isavuconazole, a more recent broad-spectrum azole, available in oral and parenteral forms, is under clinical investigation. Scedosporium spp are intrinsically resistant to AmB. While Scedosporium apiospermum is susceptible to itraconazole, voriconazole, and posaconazole, Scedosporium prolificans is considered resistant to all available antifungal agents. ${ }^{39,40}$ Limited data are available to support the optimal antifungal therapy against Acremonium spp. Clinically relevant species of Acremonium, Alternaria, Cladosporium and Exophiala have demonstrated high minimal inhibitory concentrations for AmB. ${ }^{41}$

\section{Safety and tolerance: L-AmB}

Conventional amphotericin B (AmB-deoxycholate [AmB-D]) was considered the gold standard of antifungal treatment for many decades. Infusion-related reactions and dose-dependent nephrotoxicity are major limitations of this drug. ${ }^{21}$ Indeed, these side effects are associated with increased morbidity in immunocompromised patients. ${ }^{42}$ Other toxicities reported with the use of AmB-D include local thrombophlebitis, nausea and vomiting, and anemia. Less frequently, hepatotoxicity (ie, transient elevation of transaminase levels and acute liver failure) has been associated with the use of AmB-D. ${ }^{42}$

In general, all lipid formulations of $\mathrm{AmB}$ have been associated with reduced nephrotoxicity compared with AmB-D. ${ }^{21,43,44}$ White et al reported a randomized, double-blind multicenter superiority trial in which $\mathrm{ABCD}(4 \mathrm{mg} / \mathrm{kg} / \mathrm{d})$ was compared with AmB-D (0.8 mg/kg/d) for the empiric management of febrile neutropenia. ${ }^{43}$ Therapeutic response was similar $(43 \%-50 \%)$, but renal dysfunction was less likely to develop and occurred later in ABCD recipients $(P<0.001$ for both parameters). Infusion-related hypoxia and chills were more common in ABCD recipients than AmB-D recipients ( $P=0.013$ and $P=0.018$, respectively). In clinical practice, in view of its frequent infusion-related intolerability, $\mathrm{ABCD}$ is not favored.

In a randomized, double-blind comparative study, Wingard et al evaluated the safety of L-AmB versus ABLC in the empirical treatment of febrile neutropenia. ${ }^{45}$ In this study, L-AmB at a dose of 3 or $5 \mathrm{mg} / \mathrm{kg} / \mathrm{d}$ was associated with significantly fewer infusion-related reactions and other toxicities (ie, nausea, vomiting) when compared to ABLC at a dose of $5 \mathrm{mg} / \mathrm{kg} / \mathrm{d}$. There was nonsignificant difference in 
the rates of hepatotoxicity, hypokalemia, and anemia between patients receiving either ABLC or L-AmB. Since the publication of Wingard et al's study, it has been widely believed that L-AmB is less nephrotoxic than ABLC. However, in a recently published study, Safdar et al conducted a metaanalysis to evaluate nephrotoxicity associated with ABLC and L-AmB. ${ }^{46}$ After adjusting for heterogeneity across the studies included in the meta-analysis, the authors found that the nephrotoxicity was similar for ABLC and L-AmB in patients receiving either drug as antifungal therapy or prophylaxis.

While all three lipid formulations of $A m B$ exhibit reduced nephrotoxicity compared with AmB-D, L-AmB appears to be the safest in terms of infusion-related reactions. Table 1 summarizes the current US Food and Drug Administration (FDA)-approved indications for the use of L-AmB and all available antifungal agents.

\section{Empirical therapy: liposomal amphotericin B (L-AmB)}

The strategy of empirical antifungal therapy has been systemically evaluated only in the setting of antibiotic-refractory fever in patients with chemotherapy-induced neutropenia. Table 2 summarizes the clinical trials of L-AmB in the empiric management of fever in neutropenic patients.

In a randomized trial by Walsh et al, L-AmB was as effective as AmB-D for the empirical therapy of fever in adult and pediatric neutropenic patients. ${ }^{47}$ In this study, 687 patients were randomized to empirical treatment with $\mathrm{L}-\mathrm{AmB}$ at a dose of $3.0 \mathrm{mg} / \mathrm{kg} / \mathrm{d}$ or AmB-D at $0.6 \mathrm{mg} / \mathrm{kg} / \mathrm{d} .{ }^{47}$ Rates of successful treatment were similar $(50.1 \%$ for patients receiving $\mathrm{L}-\mathrm{AmB}$ and $49.4 \%$ for those receiving AmB-D.) Rates of survival were also similar (93\% for L-AmB and 90\% for AmB-D). Significantly, the use of L-AmB was associated with fewer proven breakthrough IFIs (11 patients [3.2\%]) compared to patients receiving AmB-D (27 patients [7.8\%], $P=0.009$ ).

As previously discussed, Wingard et al conducted a randomized, double-blind comparative study of L-AmB (3-5 mg/kg/d) versus ABLC $(5 \mathrm{mg} / \mathrm{kg} / \mathrm{d})$ in the empirical treatment of febrile neutropenia. ${ }^{45}$ Among 244 patients included, actual response rates were $40 \%(3 \mathrm{mg} / \mathrm{kg} / \mathrm{d})$ and $42 \%$ ( $5 \mathrm{mg} / \mathrm{kg} / \mathrm{d}$ ) for L-AmB, and $33 \%$ for ABLC. While the primary endpoint in this study was the incidence of infusion-related reactions, the authors found no significant differences in successful response and survival in both adult and pediatric patients. ${ }^{45}$

With the introduction of new classes of antifungal agents, the efficacy of L-AmB for empirical therapy has been compared with that of caspofungin ${ }^{48-50}$ and voriconazole. ${ }^{51}$ Many prospective, randomized, multicenter comparative trials evaluating echinocandins have been published ${ }^{48,49,51}$ (Table 2). In these studies, efficacy assessment was based on a composite endpoint consisting of five criteria: (1) successful treatment of any baseline fungal infection, (2) absence of any breakthrough fungal infection during therapy or within 7 days after the completion of therapy, (3) survival for 7 days after the completion of therapy, (4) no premature discontinuation of study therapy because of drug-related toxicity or lack of efficacy,

Table I US Food and Drug Administration-approved indication of antifungal agents for the management of IFls

\begin{tabular}{|c|c|c|c|c|c|c|c|c|c|}
\hline \multirow[t]{2}{*}{ Indication } & \multicolumn{3}{|c|}{ Echinocandins } & \multicolumn{2}{|c|}{ Newer azoles } & \multicolumn{4}{|l|}{ Polyenes } \\
\hline & CAS & MICA & ANID & VOR & POS & AmB-D & ABLC & ABCD & L-AmB \\
\hline Empiric treatment of invasive fungal infections & YES & & & & & & & & YES \\
\hline Prophylaxis of Candida infections & & YES & & & YES* & & & & \\
\hline Prophylaxis of invasive aspergillosis & & & & & YES* & & & & \\
\hline Target therapy of invasive aspergillosis & YES & & & YES & & $\mathrm{YES}^{\dagger}$ & YES $^{++}$ & YES & YES $^{\circ}$ \\
\hline Target therapy of mucormycosis & & & & & & YES $^{\dagger}$ & YES ++ & & \\
\hline Salvage treatment of IFIs & & & & YES• & & & YES $^{++}$ & & \\
\hline Oropharyngeal candidiasis & YES & YES & YES & YES & & & $\mathrm{YES}^{++}$ & & $\mathrm{YES}^{\circ}$ \\
\hline Invasive candidiasis & YES & YES & YES & YES•• & & YES $^{\dagger}$ & YES $^{++}$ & & YES $^{\circ}$ \\
\hline
\end{tabular}

Notes: *In patients aged $\geq 13$ years, who are at high risk of developing these infections due to being severely immunocompromised, such as hematopoietic stem-cell transplant recipients with graft versus host disease or those with hematologic malignancies with prolonged neutropenia from chemotherapy; ${ }^{\S}$ in patients undergoing allogeneic hematopoietic stem-cell transplant; "infections with Scedosporium spp and Fusarium spp in patients refractory to or intolerant of other agents. "in nonneutropenic patients. ${ }^{\dagger} \mathrm{AmB}-\mathrm{D}$ is approved for the treatment of potentially life-threatening fungal infections: aspergillosis; cryptococcosis (torulosis); North American blasmomycosis; systemic candidiasis; coccidioidomycosis; histoplasmosis; and zgomycosis including mucormycosis due to susceptible species of the genera Absidia, Mucor, and Rhizopus and infections due to related susceptible species of Conidiobolus and Basidiobolus, and sporotrichosis; ${ }^{\dagger+} \mathrm{ABLC}$ approved for the treatment of invasive fungal infections in patients who are refractory to or intolerant of conventional amphotericin $B$; ${ }^{\ddagger} A B C D$ is approved for the treatment of invasive aspergillosis in patients who are refractory to or intolerant of conventional amphotericin B; ${ }^{\circ}$ L-AmB (AmBisome) is approved for the treatment of patients with Aspergillus spp, Candida spp, and/or infections due to Cryptococcus spp refractory to conventional $\mathrm{AmB}$ or in patients with marked renal impairment or when severe toxicity precludes the use of conventional AmB; also approved for the treatment of cryptococcal meningitis in human immunodeficiency virus-infected patients and visceral leishmaniasis.

Abbreviations: $A B C D$, amphotericin B colloidal dispersion; AmB-D, amphotericin B deoxycolate; ABLC, amphotericin B lipid complex; ANID, anidulafungin; CAS, caspofungin; IFIs, invasive fungal infections; L-AmB, liposomal amphotericin B; MICA, micafungin; POS, posaconazole; VOR, voriconazole. 


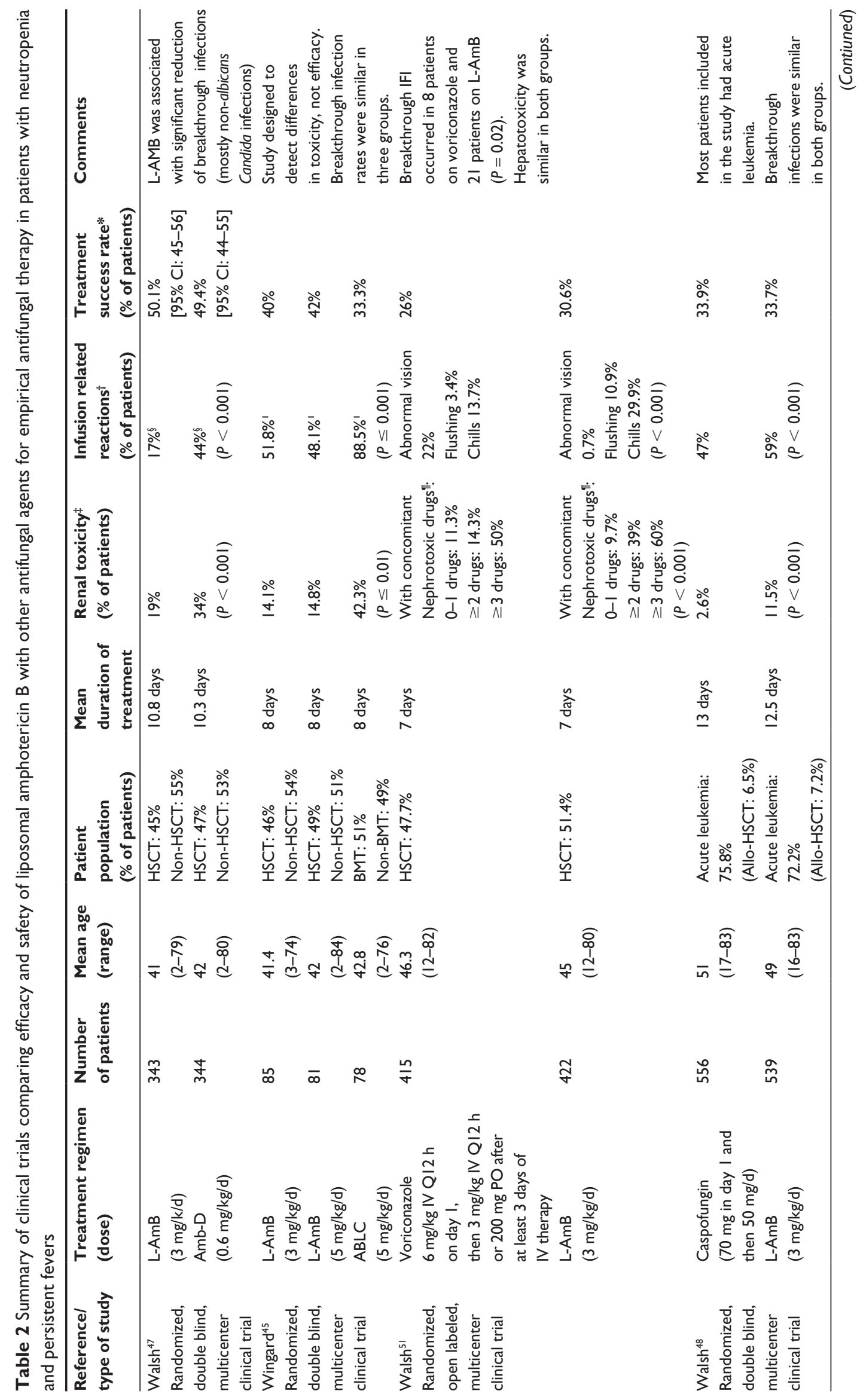




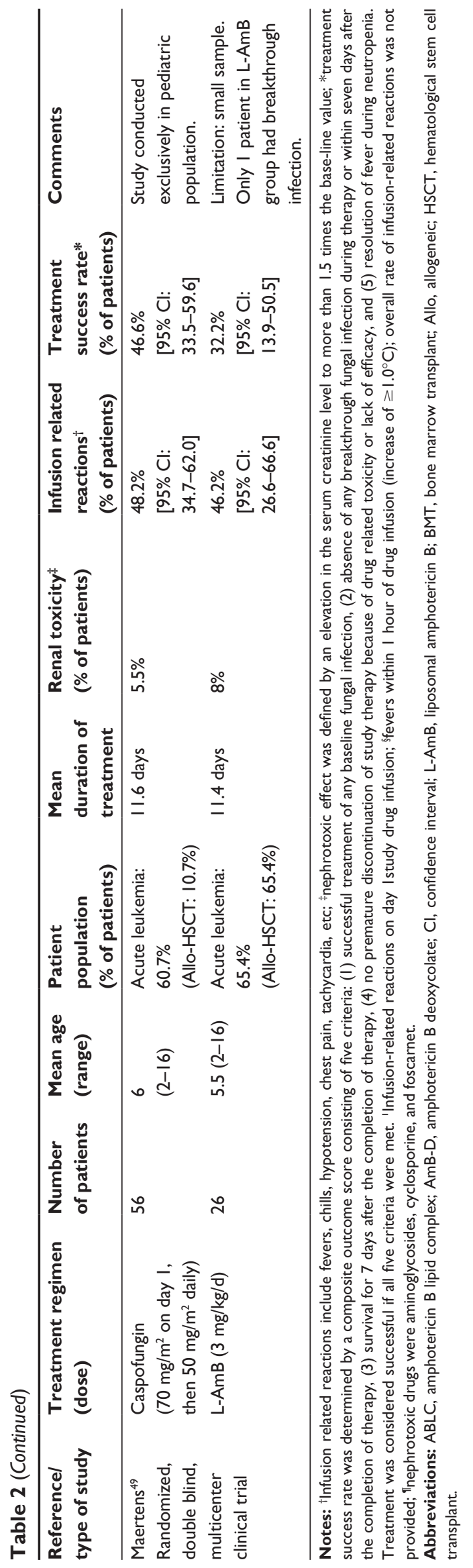

and (5) resolution of fever during neutropenia. Treatment was considered successful if all five criteria were met. ${ }^{47}$

In a randomized, double-blind multicenter trial of 1095 patients with persistent fever and neutropenia, Walsh et al showed that caspofungin was as effective as L-AmB..$^{48}$ The overall success rates were $33.9 \%$ for caspofungin and $33.7 \%$ for L-AmB (95.2\% confidence interval: -5.6 to 6.0$)$ caspofungin improved survival $(92.6 \%$ and $89.2 \%$ for caspofungin and L-AmB, respectively; $P=0.05$ ) and response rates in patients with IFIs $(51.9 \%$ vs $25.9 \% ; P=0.04)$. Breakthrough fungal infections were similar in the two groups. Also resolution of fever during the period of neutropenia was comparable. Fewer patients in the caspofungin group encountered nephrotoxicity and there were fewer infusion-related events or premature discontinuations of therapy. The authors concluded caspofungin to be as effective as L-AmB.

More recently, Maertens et al reported the results of a randomized, double-blind multicenter trial comparing empirical therapy with caspofungin versus L-AmB in pediatric patients. ${ }^{49}$ In this study, patients were randomized to receive caspofungin (70 mg loading dose on day 1 followed by $50 \mathrm{mg} / \mathrm{d}$ ) or L-AmB $(3 \mathrm{mg} / \mathrm{kg} / \mathrm{d})$. Although the main endpoint of this study was safety, clinical efficacy was also evaluated. Overall success rates were similar across treatment groups (46.4\% and $32 \%$ for caspofungin and L-AmB, respectively).

Walsh et al conducted a randomized, nonblinded multicenter trial among febrile neutropenic patients comparing empirical antifungal therapy with L-AmB versus voriconazole. ${ }^{51}$ In this study, voriconazole failed to meet the prespecified criteria for noninferiority to L-AmB. A total of 837 patients (415 assigned to voriconazole and 422 to L-AmB) were evaluated for success of treatment. Based upon prespecified criteria, voriconazole was inferior to L-AmB with overall success rates of $26 \%$ with voriconazole and $30.6 \%$ with L-AmB (95\% confidence interval: -10.6 to 1.6). Importantly, however, documented breakthrough IFIs occurred less frequently among voriconazole recipients - eight patients $(1.9 \%)$ in the voriconazole group versus 21 patients (5\%) in the L-AmB group $(P=0.02)$. After stratifying for risk, overall success rates were $32 \%$ and $30 \%$ for high-risk patients receiving voriconazole and L-AmB, respectively. The authors concluded that voriconazole is a suitable alternative to L-AmB for empiric antifungal therapy in patients with neutropenia and persistent fever.

Because of its broad spectrum of activity, rarely documented resistance, and long track record of clinical efficacy, AmB-D has been the standard choice for the empirical treatment in patients with persistent fever and 
neutropenia for many decades. However, antifungal agents that have become available over the past 15 years are less toxic and are as effective as AmB-D in the empiric treatment of IFIs. ${ }^{45,47-51}$ Indeed, based on the data discussed, L-AmB and caspofungin have now been approved by the FDA for the empiric antifungal therapy in febrile neutropenic patients. ${ }^{52,53}$

All echinocandins are safe, well tolerated, and have a similar spectrum of antifungal activity. However, FDA-approved indications of these drugs differ. ${ }^{52,53}$ Caspofungin is approved for empirical therapy of febrile neutropenia and for the treatment of invasive aspergillosis in patients refractory of or intolerant to other approved drugs. ${ }^{53}$ Micafungin, however, has been approved for antifungal prophylaxis in stem-cell transplantation. Limited data suggest that the safety and efficacy of caspofungin and micafungin are comparable in the empiric treatment of invasive aspergillosis, but micafungin has not been licensed for this use. ${ }^{54}$ Similarly, despite the widespread use of voriconazole as empiric therapy and prophylaxis of IFIs, voriconazole is not FDA approved for these indications. ${ }^{55}$ Posaconazole, based on available data, has been approved for the prophylaxis of invasive Aspergillus and Candida infections in high-risk, severely immunocompromised adult patients. ${ }^{55}$

In summary, the three lipid formulations of AmB have demonstrated similar efficacy and fewer adverse events compared with conventional amphotericin B deoxycholate. Amphotericin B colloidal dispersion, in view of its unacceptably high frequency of infusion-related toxicities similar to amphotericin B deoxycholate, is no longer favored. L-AmB has fewer infusion-related adverse reactions as compared with ABLC. Both L-AmB and ABLC appear to have similar nephrotoxic potential.

$\mathrm{L}-\mathrm{AmB}$ has been rigorously evaluated in the empiric treatment of patients with neutropenia and antibiotic-refractory fever. The drug appears safe as well as effective. Besides neutropenic fever, empiric therapy may be required in some nonneutropenic situations as well. For example, allogeneic stem-cell recipients with graft versus host disease may require empiric antifungal therapy in the setting of undiagnosed, nodular pulmonary infiltrates. Although not clinically proven, L-AmB may be an appropriate empiric choice in such situations, particularly in the background of prophylactic use of broad-spectrum azoles. During the last decade, many thought that amphotericin B-deoxycholate and its lipid forms would become obsolete in view of the recently released, less-toxic azoles and echinocandins. That has not turned out to be so. Despite the fact that amphotericin B is an "old" drug, $\mathrm{L}-\mathrm{AmB}$ plays and will continue to play an important role in the empiric antifungal management of patients at high risk for invasive fungal infections.

\section{Disclosure}

The authors report no conflicts of interest in this work.

\section{References}

1. Mohr J, Johnson M, Cooper T, Lewis JS, Ostrosky-Zeichner L. Current options in antifungal pharmacotherapy. Pharmacotherapy. 2008;28(5):614-645.

2. Miceli MH, Díaz JA, Lee SA. Emerging opportunistic yeast infections. Lancet Infect Dis. 2011;11:142-151.

3. Miceli MH, Lee SA. Emerging moulds: epidemiological trends and antifungal resistance. Mycoses. 2011;54(6):e666-e678.

4. Borman AM, Petch R, Linton CJ, Palmer MD, Bridge PD, Johnson EM. Candida nivariensis, an emerging pathogenic fungus with multidrug resistance to antifungal agents. J Clin Microbiol. 2008;46(3):933-938.

5. Snydman DR. Shifting patterns in the epidemiology of nosocomial Candida infections. Chest. 2003;123(5 Suppl):500S-503S.

6. Pfaller MA, Diekema DJ, Gibbs DL, et al. Results from the ARTEMIS DISK Global Antifungal Surveillance Study, 1997-2005. An 8.5-year analysis of susceptibilities of Candida species and other yeast species to fluconazole and voriconazole by CLSI standardized disk diffusion testing. J Clin Microbiol. 2007;45(6):1735-1745.

7. Baddley JW, Moser SA. Emerging fungal resistance. Clin Lab Med 2004;24(3):721-735, vii.

8. Sabatelli F, Patel R, Mann PA, et al. In vitro activities of posaconazole, fluconazole, itraconazole, voriconazole, and amphotericin B against a large collection of clinically important molds and yeasts. Antimicrob Agents Chemother. 2006;50(6):2009-2015.

9. Imhof A, Balajee SA, Fredricks DN, Englund JA, Marr KA. Breakthrough fungal infections in stem cell transplant recipients receiving voriconazole. Clin Infect Dis. 2004;39(5):743-746.

10. Kobayashi K, Kami M, Murashige N, Kishi Y, Fujisaki G, Mitamura T. Breakthrough zygomycosis during voriconazole treatment for invasive aspergillosis. Haematologica. 2004;89(11):ECR42.

11. Marty FM, Cosimi LA, Baden LR. Breakthrough zygomycosis after voriconazole treatment in recipients of hematopoietic stem-cell transplants. N Engl J Med. 2004;350(9):950-952.

12. Procop GW, Roberts GD. Emerging fungal diseases: the importance of the host. Clin Lab Med. 2004;24(3):691-719, vi-vii.

13. Ascioglu S, Rex JH, de Pauw B, et al. Defining opportunistic invasive fungal infections in immunocompromised patients with cancer and hematopoietic stem cell transplants: an international consensus. Clin Infect Dis. 2002;34(1):7-14.

14. Chandrasekar P. Invasive mold infections: recent advances in management approaches. Leuk Lymphoma. 2009;50(5):703-715.

15. Ruhnke M, Bohme A, Buchheidt D, et al. Diagnosis of invasive fungal infections in hematology and oncology - guidelines from the Infectious Diseases Working Party in Haematology and Oncology of the German Society for Haematology and Oncology (AGIHO). Ann Oncol. Epub 2011 Sep 23.

16. Freifeld AG, Bow EJ, Sepkowitz KA, et al. Clinical practice guideline for the use of antimicrobial agents in neutropenic patients with cancer: 2010 update by the infectious diseases society of america. Clin Infect Dis. 2011;52(4):e56-e93.

17. Ghannoum MA, Rice LB. Antifungal agents: mode of action, mechanisms of resistance, and correlation of these mechanisms with bacterial resistance. Clin Microbiol Rev. 1999;12(4):501-517.

18. Sucher AJ, Chahine EB, Balcer HE. Echinocandins: the newest class of antifungals. Ann Pharmacother. 2009;43(10):1647-1657.

19. Walsh TJ, Driscoll T, Milligan PA, et al. Pharmacokinetics, safety, and tolerability of voriconazole in immunocompromised children. Antimicrob Agents Chemother. 2010;54(10):4116-4123. 
20. Moen MD, Lyseng-Williamson KA, Scott LJ. Liposomal amphotericin B: a review of its use as empirical therapy in febrile neutropenia and in the treatment of invasive fungal infections. Drugs. 2009;69(3): 361-392.

21. Dupont B. Overview of the lipid formulations of amphotericin B. J Antimicrob Chemother. 2002;49(Suppl 1):31-36.

22. Anaissie E, Paetznick V, Proffitt R, Adler-Moore J, Bodey GP. Comparison of the in vitro antifungal activity of free and liposome-encapsulated amphotericin B. Eur J Clin Microbiol Infect Dis. 1991;10(8): 665-668.

23. Hawkins JL, Baddour LM. Candida lusitaniae infections in the era of fluconazole availability. Clin Infect Dis. 2003;36(2):e14-e18.

24. Krcmery V, Barnes AJ. Non-albicans Candida spp causing fungaemia: pathogenicity and antifungal resistance. J Hosp Infect. 2002;50(4): 243-260.

25. Colombo AL, Melo AS, Crespo Rosas RF, et al. Outbreak of Candida rugosa candidemia: an emerging pathogen that may be refractory to amphotericin B therapy. Diagn Microbiol Infect Dis. 2003;46(4): $253-257$.

26. Araujo Ribeiro M, Alastruey-Izquierdo A, Gomez-Lopez A, RodriguezTudela JL, Cuenca-Estrella M. Molecular identification and susceptibility testing of Trichosporon isolates from a Brazilian hospital. Rev Iberoam Micol. 2008;25(4):221-225.

27. Pfaller MA, Diekema DJ. Rare and emerging opportunistic fungal pathogens: concern for resistance beyond Candida albicans and Aspergillus fumigatus. J Clin Microbiol. 2004;42(10):4419-4431.

28. Baddley JW, Pappas PG, Smith AC, Moser SA. Epidemiology of Aspergillus terreus at a university hospital. J Clin Microbiol. 2003;41(12): 5525-5529.

29. Iwen PC, Rupp ME, Langnas AN, Reed EC, Hinrichs SH. Invasive pulmonary aspergillosis due to Aspergillus terreus: 12-year experience and review of the literature. Clin Infect Dis. 1998;26(5):1092-1097.

30. Sutton DA, Sanche SE, Revankar SG, Fothergill AW, Rinaldi MG. In vitro amphotericin $\mathrm{B}$ resistance in clinical isolates of Aspergillus terreus, with a head-to-head comparison to voriconazole. J Clin Microbiol. 1999;37(7):2343-2345.

31. Verweij PE, van den Bergh MF, Rath PM, de Pauw BE, Voss A, Meis JF. Invasive aspergillosis caused by Aspergillus ustus: case report and review. J Clin Microbiol. 1999;37(5):1606-1609.

32. Iwen PC, Rupp ME, Bishop MR, et al. Disseminated aspergillosis caused by Aspergillus ustus in a patient following allogeneic peripheral stem cell transplantation. J Clin Microbiol. 1998;36(12):3713-3717.

33. Panackal AA, Imhof A, Hanley EW, Marr KA. Aspergillus ustus infections among transplant recipients. Emerg Infect Dis. 2006;12(3): 403-408.

34. Balajee SA, Gribskov JL, Hanley E, Nickle D, Marr KA. Aspergillus lentulus sp. nov, a new sibling species of A. fumigatus. Eukaryot Cell. 2005;4(3):625-632.

35. Balajee SA, Weaver M, Imhof A, Gribskov J, Marr KA. Aspergillus fumigatus variant with decreased susceptibility to multiple antifungals. Antimicrob Agents Chemother. 2004;48(4):1197-1203.

36. Pujol I, Guarro J, Gené J, Sala J. In-vitro antifungal susceptibility of clinical and environmental Fusarium spp strains. J Antimicrob Chemother. 1997;39(2):163-167.

37. Denning DW. Echinocandin antifungal drugs. Lancet. 2003;362(9390): 1142-1151.

38. van Burik JA, Hare RS, Solomon HF, Corrado ML, Kontoyiannis DP. Posaconazole is effective as salvage therapy in zygomycosis: a retrospective summary of 91 cases. Clin Infect Dis. 2006;42(7):e61-e65.
39. Cuenca-Estrella M, Ruiz-Díez B, Martinez-Suárez JV, Monzón A, Rodríguez-Tudela JL. Comparative in-vitro activity of voriconazole (UK-109,496) and six other antifungal agents against clinical isolates of Scedosporium prolificans and Scedosporium apiospermum. $J$ Antimicrob Chemother. 1999;43(1):149-151.

40. Walsh TJ, Peter J, McGough DA, Fothergill AW, Rinaldi MG, Pizzo PA. Activities of amphotericin $\mathrm{B}$ and antifungal azoles alone and in combination against Pseudallescheria boydii. Antimicrob Agents Chemother. 1995;39(6):1361-1364.

41. Perdomo H, Sutton DA, Garcia D, et al. Spectrum of clinically relevant Acremonium species in the United States. J Clin Microbiol. 2011;49(1): 243-256.

42. Gallis HA, Drew RH, Pickard WW. Amphotericin B: 30 years of clinical experience. Rev Infect Dis. 1990;12(2):308-329.

43. White MH, Bowden RA, Sandler ES, et al. Randomized, double-blind clinical trial of amphotericin B colloidal dispersion vs amphotericin B in the empirical treatment of fever and neutropenia. Clin Infect Dis. 1998;27(2):296-302.

44. Ostrosky-Zeichner L, Marr KA, Rex JH, Cohen SH. Amphotericin B: time for a new "gold standard". Clin Infect Dis. 2003;37(3):415-425.

45. Wingard JR, White MH, Anaissie E, Raffalli J, Goodman J, Arrieta A. A randomized, double-blind comparative trial evaluating the safety of liposomal amphotericin B versus amphotericin B lipid complex in the empirical treatment of febrile neutropenia. L Amph/ABLC Collaborative Study Group. Clin Infect Dis. 2000;31(5):1155-1163.

46. Safdar A, Ma J, Saliba F, et al. Drug-induced nephrotoxicity caused by amphotericin B lipid complex and liposomal amphotericin B: a review and meta-analysis. Medicine (Baltimore). 2010;89(4):236-244.

47. Walsh TJ, Finberg RW, Arndt C, et al. Liposomal amphotericin B for empirical therapy in patients with persistent fever and neutropenia. National Institute of Allergy and Infectious Diseases Mycoses Study Group. N Engl J Med. 1999;340(10):764-771.

48. Walsh TJ, Teppler H, Donowitz GR, et al. Caspofungin versus liposomal amphotericin B for empirical antifungal therapy in patients with persistent fever and neutropenia. N Engl J Med. 2004;351(140):1391-1402.

49. Maertens JA, Madero L, Reilly AF, et al. A randomized, double-blind, multicenter study of caspofungin versus liposomal amphotericin B for empiric antifungal therapy in pediatric patients with persistent fever and neutropenia. Pediatr Infect Dis J. 2010;29(5):415-420.

50. Ellis M, Frampton C, Joseph J, et al. An open study of the comparative efficacy and safety of caspofungin and liposomal amphotericin B in treating invasive fungal infections or febrile neutropenia in patients with haematological malignancy. J Med Microbiol. 2006;55(Pt 10): 1357-1365.

51. Walsh TJ, Pappas P, Winston DJ, et al. Voriconazole compared with liposomal amphotericin B for empirical antifungal therapy in patients with neutropenia and persistent fever. $N$ Engl J Med. 2002;346(4): 225-234.

52. USA FDA Regulations. http://www.fda.gov/drugs/default.htm.

53. Chen SC, Slavin MA, Sorrell TC. Echinocandin antifungal drugs in fungal infections: a comparison. Drugs. 2011;71(1):11-41.

54. Kubiak DW, Bryar JM, McDonnell AM, et al. Evaluation of caspofungin or micafungin as empiric antifungal therapy in adult patients with persistent febrile neutropenia: a retrospective, observational, sequential cohort analysis. Clin Ther. 2010;32(4):637-648.

55. Waknine Y. FDA approvals. Avelox, Avandaryl, Trileptal. Available at: http://www.medscape.com/viewarticle/518278?rss. Accessed February 11, 2006 [cited 2011 Oct 24].
Infection and Drug Resistance

\section{Publish your work in this journal}

Infection and Drug Resistance is an international, peer-reviewed openaccess journal that focuses on the optimal treatment of infection (bacterial, fungal and viral) and the development and institution of preventive strategies to minimize the development and spread of resistance. The journal is specifically concerned with the epidemiology of antibiotic
Dovepress

resistance and the mechanisms of resistance development and diffusion in both hospitals and the community. The manuscript management system is completely online and includes a very quick and fair peerreview system, which is all easy to use. Visit http://www.dovepress.com/ testimonials.php to read real quotes from published authors. 